\title{
Determination of intergalactic magnetic fields from gamma ray data
}

\author{
Warren Essey ${ }^{\mathrm{a}}$, Shin'ichiro Ando ${ }^{\mathrm{b}}$, Alexander Kusenko ${ }^{\mathrm{a}, \mathrm{c}}$ \\ ${ }^{a}$ Department of Physics and Astronomy, University of California, Los Angeles, CA \\ 90095-1547, USA \\ ${ }^{b}$ California Institute of Technology, Mail Code 350-17, Pasadena, CA 91125, USA \\ ${ }^{c} I P M U$, University of Tokyo, Kashiwa, Chiba 277-8568, Japan
}

\begin{abstract}
We report a measurement of intergalactic magnetic fields using combined data from Atmospheric Cherenkov Telescopes and Fermi Gamma-Ray Space Telescope, based on the spectral data alone. If blazars are assumed to produce both gamma rays and cosmic rays, the observed spectra are not sensitive to the intrinsic spectrum of the source, because, for a distant blazar, secondary photons produced along the line of sight dominate the signal. In this case, we find $1 \times 10^{-17} \mathrm{G}<B<3 \times 10^{-14} \mathrm{G}$. If one excludes the cosmic-ray component, the $10^{-17} \mathrm{G}$ lower limit remains, but the upper limit depends on the spectral properties of the source. We present the allowed ranges for a variety of model parameters.
\end{abstract}

Intergalactic magnetic fields (IGMFs) play an important role in many astrophysical processes and may offer a new window on the cosmology in the early universe, but the size and origin of these fields are still poorly understood [1]. Until recently, only the upper limits of $10^{-9} \mathrm{G}$ were inferred from the observational data [2]. One can measure IGMFs using gamma-ray observations, for example, using time delays [3], or by searching extended halos around the point objects [4, 5]. Here we employ an independent and complementary approach to obtain new upper and lower limits on IGMFs based on the spectra of three blazars observed by HESS, for which there also exist upper limits from Fermi Gamma-Ray Space Telescope. We have calculated the spectra numerically, and we use the goodness of fit to the data as the means to infer the average values of IGMFs. Blazar spectra have been used to set lower bounds on the IGMF [6, 7, 8, 9]. However, in 
each of Refs. [6, 7, 8], only one EBL model and a single power law injection spectrum were considered, and the cosmic ray contribution was not included in Refs. [6, 7, 8, 9], while it can be the dominant contribution for distant sources [10, 11, 12]. Our analysis is different from previous work in that we have scanned over a very wide range of model parameters, and we included the cosmic-ray component.

AGN are known to be sources of very high energy (VHE) gamma rays; they are also believed to produce cosmic rays [13, 14]. There is growing evidence that both primary gamma rays emitted at the source and secondary gamma rays produced by cosmic rays along the line of sight contribute to the observed signals [10, 11, 12]. We, therefore, consider two cases: (i) pure gamma-ray emission at the source, and (ii) mixed gamma-ray and cosmic ray emission. In the case (i) the intrinsic source spectra determine the observed spectra. If, however, the cosmic-ray contribution (ii) is included, the observed gamma-ray spectra are remarkably independent of the intrinsic gamma-ray or cosmic-ray spectra at the source. The observed spectrum is determined almost entirely by the shape (but not the overall normalization) of extragalactic background light (EBL) spectrum in case (ii), and the overall agreement of such a prediction with observed spectra of distant blazars can be considered evidence of cosmic ray contribution, and, therefore, of cosmic ray acceleration in AGN [10, 11, 12]. Nevertheless, in what follows, we will consider both possibilities (i) and (ii). In the case (i), most of the observed secondary gamma rays are produced within tens of megaparsecs from the source, and our measurement reflects IGMFs closer to the source. In the case (ii), the field strength we infer corresponds to equally weighted averaging along the line of sight.

To probe IGMF we constructed a detailed Monte Carlo simulation that accurately tracks the effects of both electromagnetic showers and cosmicray propagation and interactions. Included in the simulation are both pion photo-production and proton pair production for cosmic rays and pair production and inverse Compton scattering for gamma rays and electrons. Any relevant secondary decays such as neutron and pion decays were also included. Deflections due to the IGMF are tracked with both momentum and position vectors stored at all points of the propagation. To construct observed spectra, the position and momentum vectors of gamma rays at the $z=0$ surface are used to calculate the image size. Individual gamma-rays are tracked to the instrument, and their arrival direction further scattered by the instrumental PSF. Any events with arrival directions remaining with 
the PSF radius are then retained. (A study of the source morphology as a function of energy can be a good diagnostic technique, but in this paper we concentrate only on the spectral analysis.) For $E_{\gamma}<100 \mathrm{GeV}$ the publicly available Fermi PSF was used for comparison and for $E_{\gamma}>100 \mathrm{GeV}$ HESS PSF was used.

Suppression of the low-energy spectra of point images depends on the mean field along the line of sight more than on the (poorly known) distribution of IGMFs. Indeed, one can use the results of Ref. [8] to compare the effects of uniform magnetic fields with those of more realistic simulations, which take into account the distribution of matter in the universe [15]. The spectrum for "MHD(x)" ("Model 3x") model, with the mean field of $100 \mathrm{fG}$ (0.1 fG), is close to that of the uniform $100 \mathrm{fG}(0.1 \mathrm{fG})$ field. One can also understand this from general arguments [10, 11, 12]. Thus, we modeled the IGMF as a characteristic strength organized into cubes of a characteristic correlation length each with a random direction. Of course, if the distribution of matter along the line of sight is known, e.g., from Lyman- $\alpha$ forest in the direction to some blazars, one can further improve our inferences of IGMFs.

From the available sources, we chose a subset based on three conditions: (a) the source must be observed at energies above $1 \mathrm{TeV}$, (b) the distance to the source must be relatively large, and (c) the source must show no temporal variability above $1 \mathrm{TeV}$. The latter condition is an necessary but not sufficient condition that secondary gamma rays dominate the observed signal, since primary gamma rays from blazars are predicted to show temporal variability. Based on these criteria, we chose three sources: 1ES 0229+200 $(z=0.14)$, 1ES1101-232 $(z=0.186)$, and 1ES0347-121 $(z=0.188)$, each of which has been observed by HESS [16, 17, 18]. Temporal variability has been measured above the $200 \mathrm{GeV}$ energy threshold of the HESS array for 1ES1101 and $1 \mathrm{ES} 0347$ [19].

We consider a range of viable EBL models. A lower limit on the EBL density is set by galaxy counts [21]. The upper limit is less known. Although gamma-ray data have been used to exclude higher EBL models such as Stecker et al. [22], it was recently pointed out that the inclusion of secondary gamma rays from VHE gamma rays and cosmic rays significantly improves the fits to the data for these "high EBL" models [12]. Therefore, we include the model of Stecker et al. [22] as the upper limit (for UV EBL). A number of other models fall between these two limits [23]. EBL models with a higher level of UV background radiation are difficult to reconcile with 

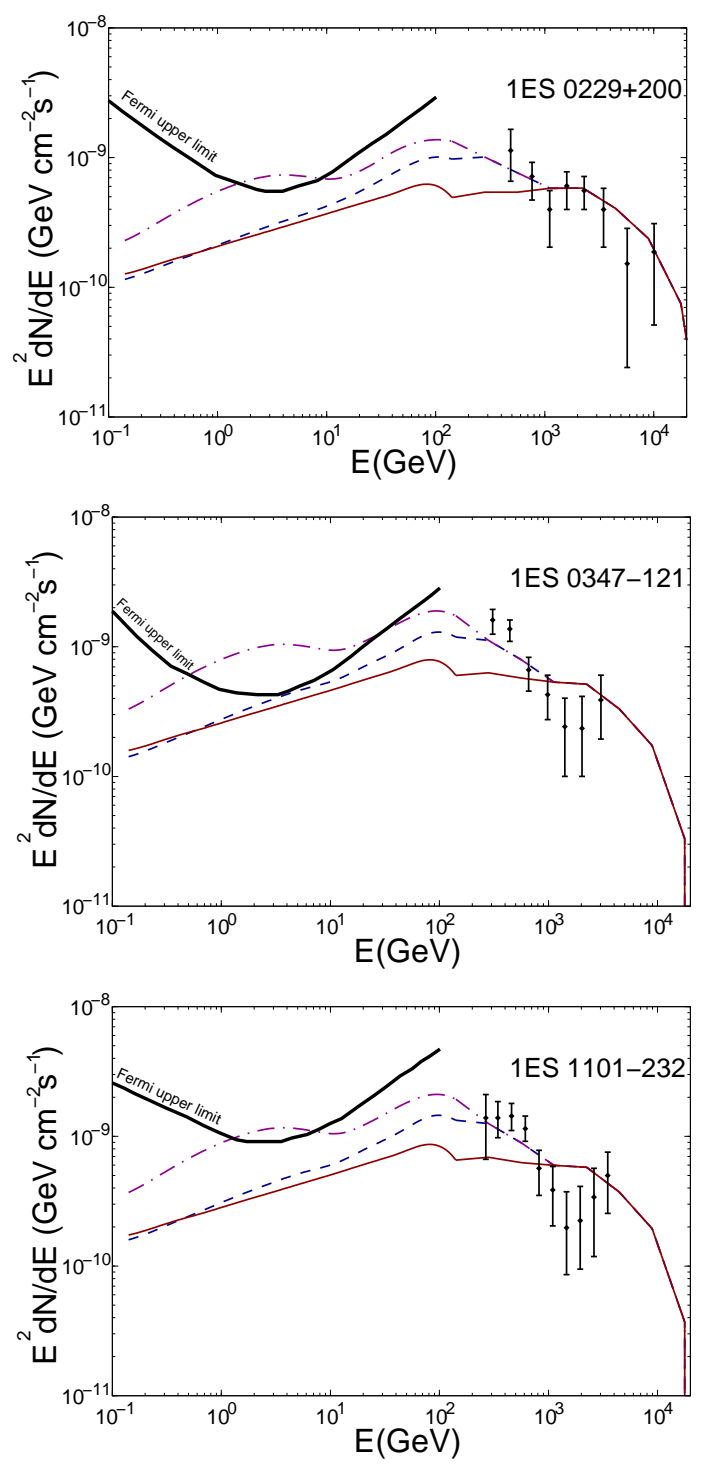

Figure 1: Photon spectra calculated numerically and normalized to fit HESS data points for $1 \mathrm{ES} 0229+200(z=0.14)$ [6], 1ES1101-232 $(z=0.186)$ [17], and 1ES0347-121 $(z=$ 0.188) [18]. The Fermi upper limits at lower energy (thick solid line) were derived from the data in Ref. [6]. The three model predictions are for primary photons and $B=10^{-18} \mathrm{G}$ (upper, dash-dotted line), $B=10^{-15} \mathrm{G}$ (middle, dashed line), and $\mathrm{B}=10^{-13} \mathrm{G}$ (lower, thin solid line). The PSF of Fermi is folded into the signal below $100 \mathrm{GeV}$ and the PSF of HESS is folded into the signal above $100 \mathrm{GeV}$. Here we assumed gamma-ray spectrum at the source $\propto E^{-1.75}$ with a $100 \mathrm{TeV}$ high-energy cutoff. 
a limit based on gamma-ray burst data [24].

We shall first discuss the case of primary gamma rays only. Following most recent papers on IGMFs, we will assume that the blazar spectra follow a power law $d N / d E \sim \mathrm{E}^{-\Gamma}$ with $\Gamma \geq 1.5$ [25]. However harder spectra are also possible [26]. Furthermore, it is possible that the intrinsic spectra do not conform to a single power law. Deviations from single power law may affect the limits on IGMFs derived under the assumption of a power-law spectrum. We consider the range $\Gamma=0.5-2$, which encompasses a broad variety of models. We also allow the high-energy cutoff of the power law spectrum to vary in a wide range, $10-10^{4} \mathrm{TeV}$.

We will consider magnetic fields ranging from $10^{-18} \mathrm{G}$ to $10^{-11} \mathrm{G}$. The IGMF correlation lengths are also not known; we assume it is in the range $0.01-1 \mathrm{Mpc}$ and use $1 \mathrm{Mpc}$ as a reference point in Fig. 1.

We fit the calculated spectrum to the data obtained by HESS Atmospheric Cherenkov Telescope (ACT) at high energy, perform statistical analysis to calculate $95 \%$ confidence intervals, and we demand that the low-energy prediction not overshoot the Fermi upper limit. The spectra obtained for 1ES $0229+200(z=0.14)$, 1ES 1101-232 $(z=0.186)$, and 1ES 0347-121 $(z=0.188)$, are shown in Fig. 1 for IGMFs in the range $10^{-18}-10^{-13} \mathrm{G}$, with a correlation length $\lambda_{c}=1 \mathrm{Mpc}$. The results for a source with intrinsic spectrum $\Gamma=1.75$, a $100 \mathrm{TeV}$ high-energy cutoff and the "low" EBL model [21], shown in Fig. 1, illustrate the effects of IGMF on the spectrum. For larger IGMFs, more of the secondary gamma rays arrive outside the cone determined by the angular resolution of the instrument, with lower energy gamma rays arriving at larger angles. This causes an energy dependent drop in the spectrum.

As one can see from comparing $10^{-15} \mathrm{G}$ and $10^{-13} \mathrm{G}$ curves in Fig. 1, the wash-out of the signal due to magnetic deflections causes a break in the spectrum at some energy, which is a function of the magnetic field. The following semi-quantitative analytical estimate may be helpful. The magnetic deflections depend on the energy [27]:

$$
\Theta_{\text {defl }} \simeq 0.1^{\circ}(1+z)^{-2}\left(\tau_{\theta} / 5\right)^{-1}\left(E_{\gamma} / 0.1 T e V\right)^{-1}\left(B / 10^{-15} G\right)
$$

for $\lambda_{\mathrm{c}} \gg \mathrm{D}_{\mathrm{e}}$, where $\tau_{\theta}$ is the optical depth of the primary gamma ray and $\mathrm{D}_{\mathrm{e}}$ the electron cooling distance. For the energies considered here, $\tau_{\theta} \propto E_{\gamma}^{-1 / 2}$, and for inverse-Compton (IC) cooling $E_{\gamma} \propto E_{\gamma 0}^{2}$, where $E_{\gamma 0}$ is the energy of the primary photon, and $n_{\gamma} \propto E_{\gamma}^{-1 / 2}$, where $n_{\gamma}$ is the number of photons 
produced from IC cooling. Thus we expect $\Theta_{\text {defl }} \propto B / E_{\gamma 0}^{3}$. Let us consider the flux of gamma rays that contribute to the point image (determined by a fixed instrumental resolution): $\Theta_{\text {defl }}<\Theta_{P S F}$ implies $E_{\gamma 0}>$ const $\times B^{1 / 3}$. Thus for an intrinsic power-law spectrum with index $\Gamma$ we expect the point flux

$$
F \propto \frac{1}{E_{\gamma}^{1 / 2}} \int_{B^{1 / 3}}^{\infty} \frac{d N}{d E_{\gamma 0}} \sim E_{\gamma}^{-1 / 2} \int_{B^{1 / 3}}^{\infty} E_{\gamma 0}^{-\Gamma} \sim \frac{1}{E_{\gamma}^{1 / 2} B^{(\Gamma-1) / 3}} .
$$

This relation implies the approximate scaling $B^{2(\Gamma-1)} E^{3}=$ const for the position of the break in the spectrum. By comparing the $10^{-15} \mathrm{G}$ and $10^{-13} \mathrm{G}$ curves in Fig. 1 we see that this approximation works reasonably well. Of course, this discussion is admittedly simplistic: the relative contributions of primary and secondary gamma rays, and some other effects were neglected. In deriving the actual limits we use the results of detailed numerical Monte Carlo for propagation, shower development, and the effects of magnetic fields.

For angular resolution of about $0.1^{\circ}$ we expect magnetic fields larger than about $1 \mathrm{fG}$ to wash out the signal observed by the ACTs, while fields lower than a fG would boost the signal in the Fermi energy range. This allows us to use the combination of ACT and Fermi data to set both upper and lower limits on the IGMF. A lower limit can be found by requiring that the IGMF is large enough to wash out enough of the GeV signal so that the observed spectrum is below the Fermi 95\% CL upper limits for the source. An upper limit can be found by requiring that the IGMF be small enough to avoid washing out the TeV signal. More specifically, we combined the ACT data for these three blazars and used 25 data points shown in Fig. 1. Then for each model with given $B$ and $\Gamma$, we have three independent parameters, i.e., normalizations (or luminosities) of the spectra of three blazars. The number of degrees of freedom of the fit is 22 . If the value of $\chi_{\min }^{2}$ for each model specified with fixed $B$ and $\Gamma$ is larger than 33.9, then such a model is acceptable at the level of only $5 \%$ probability. This way, we set excluded regions (or upper limits on $B$ ) at $95 \% \mathrm{CL}$.

Our results for pure gamma-ray sources are summarized in Fig. 2. The results depend on the choice of EBL model and high energy cutoff. We show both the "low" EBL model, based on lower limits from galaxy counts, and the "high" EBL model [22], as well as high-energy cutoff values of $20 \mathrm{TeV}$ and $100 \mathrm{TeV}$.

Let us now include the the cosmic ray component [10, 11, 12]. Now the observed gamma-ray spectrum does not depend on the intrinsic spectrum of 
gamma rays, and it is also practically independent of the spectral properties of cosmic rays, such as the high energy cutoff and the cosmic ray spectral index (under some very mild assumptions) [10, 11, 12]. Therefore, instead of the exclusion regions shown in Fig. 2, we find the following bounds (for $\left.\lambda_{c}=1 \mathrm{Mpc}\right)$ :

$$
\begin{aligned}
& 3 \times 10^{-16} \mathrm{G}<B<3 \times 10^{-14} \mathrm{G}(\text { High EBL) } \\
& 1 \times 10^{-17} \mathrm{G}<B<8 \times 10^{-16} \mathrm{G}(\text { Low EBL) }
\end{aligned}
$$

Of course it is possible, and likely, that the observed spectrum is a combination of secondary particles from gamma rays and cosmic rays emitted by the source. In this case the most conservative allowed range for IGMF would be the union of the gamma-ray and cosmic-ray allowed ranges.

The limits in Fig. 2 imply $\Gamma \leq 1.8$ for the set of blazars we chose, which is in good agreement with mean spectral index of 1.9 reported by Fermi for nearby sources.

The limits presented in Fig. 2 are subject to some caveats. First, we have assumed that our three sources had similar intrinsic spectra, which need not be the case. Second, as discussed above, the likely contribution of protons along the line of sight [10, 11, 12] can reduce the limits to the modelindependent form shown in Eq. 1. Finally, time variability of the primary gamma ray sources can affect our conclusions [9].

Following the insightful discussion of Dermer et al. [9], it can be shown that the typical time delays for secondary photons are

$$
\Delta t \simeq 2 \times 10^{6} \lambda_{100}\left(\frac{B_{-15}}{E_{\gamma} / 10 \mathrm{GeV}}\right)^{2} \text { yrs }
$$

where $\lambda_{100} \sim 1$ for the Stecker et al EBL model, $B_{-15}$ is the IGMF in units of $10^{-15} \mathrm{G}$. If the source is active for a timescale that is shorter than these delays, the observed signal is suppressed by a factor $\left(t_{\text {active }} / \Delta t\right)$ where $t_{\text {active }}$ is the time that the source was active for. This suppression mimics the effects of a stronger magnetic field, and, therefore, the predicted limit on the IGMF would be suppressed by the a factor

$$
\kappa \simeq\left(\frac{t_{\text {active }}}{2 \times 10^{6} \mathrm{yrs}}\right)^{1 / 2}\left(\frac{10^{-15} \mathrm{G}}{B_{\text {limit }}}\right)\left(\frac{E_{\gamma}}{10 \mathrm{GeV}}\right),
$$

where $B_{\text {limit }}$ is either the upper or lower limit shown in Fig. 2. 

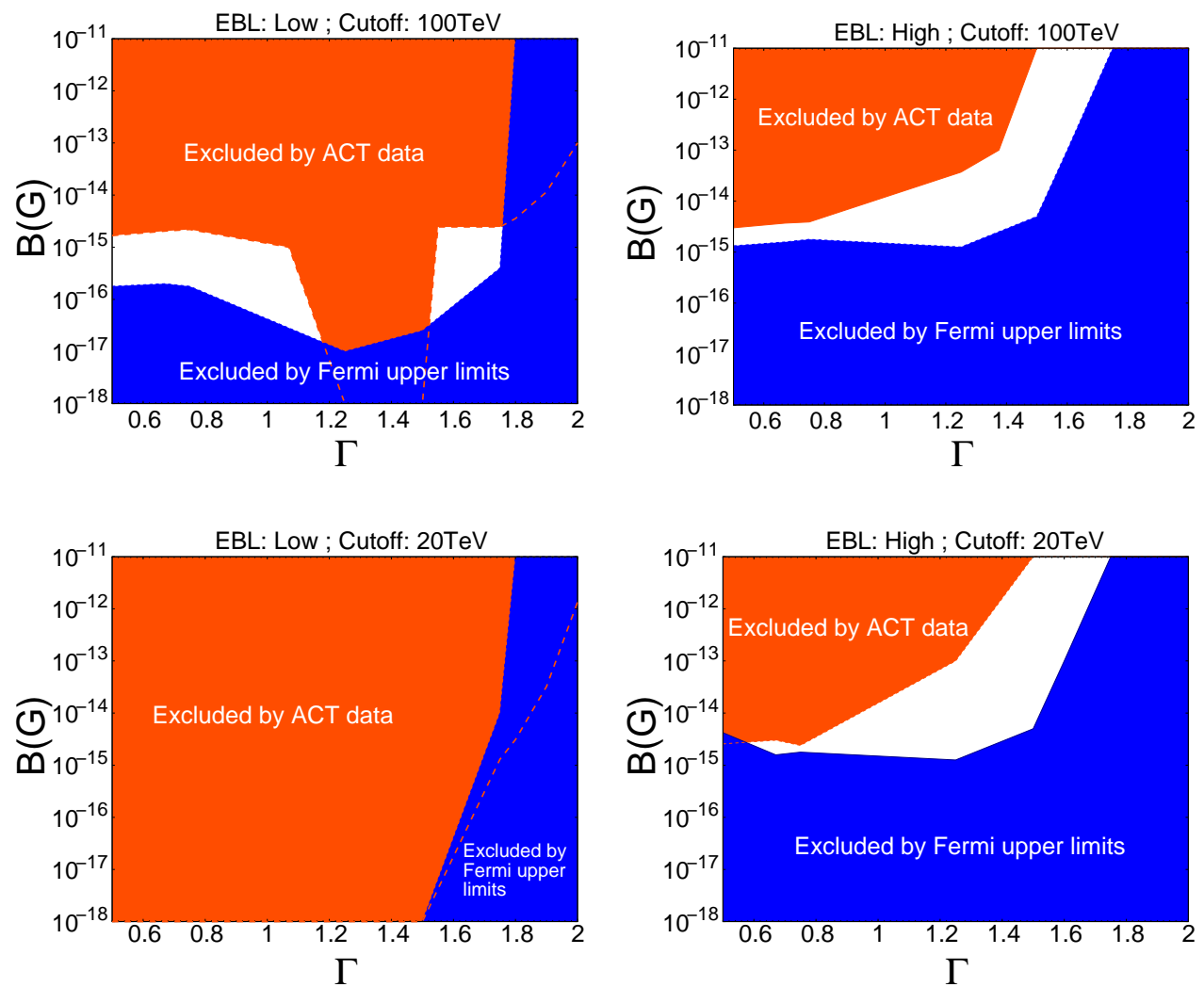

Figure 2: Limits on the magnitude of the IGMF and intrinsic spectral index based on the combined fit for spectra of 1ES 0229+200, 1ES 1101-232, and 1ES 0347-121, assuming that only gamma rays are produced at the source (no cosmic ray contribution). Shaded regions are excluded at 95\% CL from either Fermi or ACT data. The limits are shown for "low" and "high" EBL models and for two values of the high-energy cutoff: $100 \mathrm{TeV}$ and $20 \mathrm{TeV}$. IGMF correlation length is assumed to be $1 \mathrm{Mpc}$. 
The observations of 1ES $0229+100$ by HESS [16] and VERITAS [20] were separated by roughly 3 years and show no evidence of significant variability. Variability may be present during the times when the source was not observed, or at a level below the instrumental sensitivity. We assume $t_{\text {active }} \sim 3$ yrs as a lower limit on the time the source was active. The most restrictive energy for the lower limits is near $E_{\gamma} \sim 10 \mathrm{GeV}$ and most of the lower limits fall in the range $10^{-15}-10^{-17} \mathrm{G}$ which means the most conservative lower limits would be suppressed by a factor of $10-10^{3}$. For upper limits, the most restrictive energy is at $E_{\gamma} \sim 1 \mathrm{TeV}$ and since most upper limits fell into the range $10^{-13}-10^{-15} \mathrm{G}$ we expect the upper limits to also be lowered by roughly $10-10^{3}$. Thus, the overall effect of including a short activity time is to lower the $95 \%$ confidence intervals shown in Fig. 2 and our robust $10^{-17} \mathrm{G}$ lower limit would be lowered to $10^{-18} \mathrm{G}$ in the most conservative case.

It is important to distinguish between the time scale of the source activity and short-term variability. A variability of the source would not lead to a suppression discussed above because signals of variable activity would undergo significant delays in the IGMF and would be observed as a constant source with the time averaged luminosity of the flares. Thus, if the source is active on a timescale of $10^{6} \mathrm{yrs}$, the limits in Fig. 2 are valid, even in the case of variability on shorter time scales.

The effect the source activity-dormancy time scale is much weaker in the case of the cosmic ray limits, because the delays in the stronger magnetic fields near the source can wash out variability on even longer time scales. The magnetic fields within the host galaxies containing source are likely to be of the order of $1 \mu G$ which can lead to significant delays. Fig. 3 shows the delays for a $100 \mathrm{kpc}$ wide source of $10^{10} \mathrm{GeV}$ protons with $B=1 \mu \mathrm{G}$ and $l_{c}=0.1 \mathrm{kpc}$. It can be seen that the delays are significantly longer than the observed variability of blazars and thus we expect any intrinsic variability to be washed out. Such a source appears as a constant, long-lived source, and any time delays incurred in the host galaxy are not measurable. Of course, there is a significant uncertainty in the magnetic field distribution around the source, in particular, in the direction of the jet, and the values assumed here are taken solely to illustrate the idea.

The effect of the source variability would be to damp the observed power 


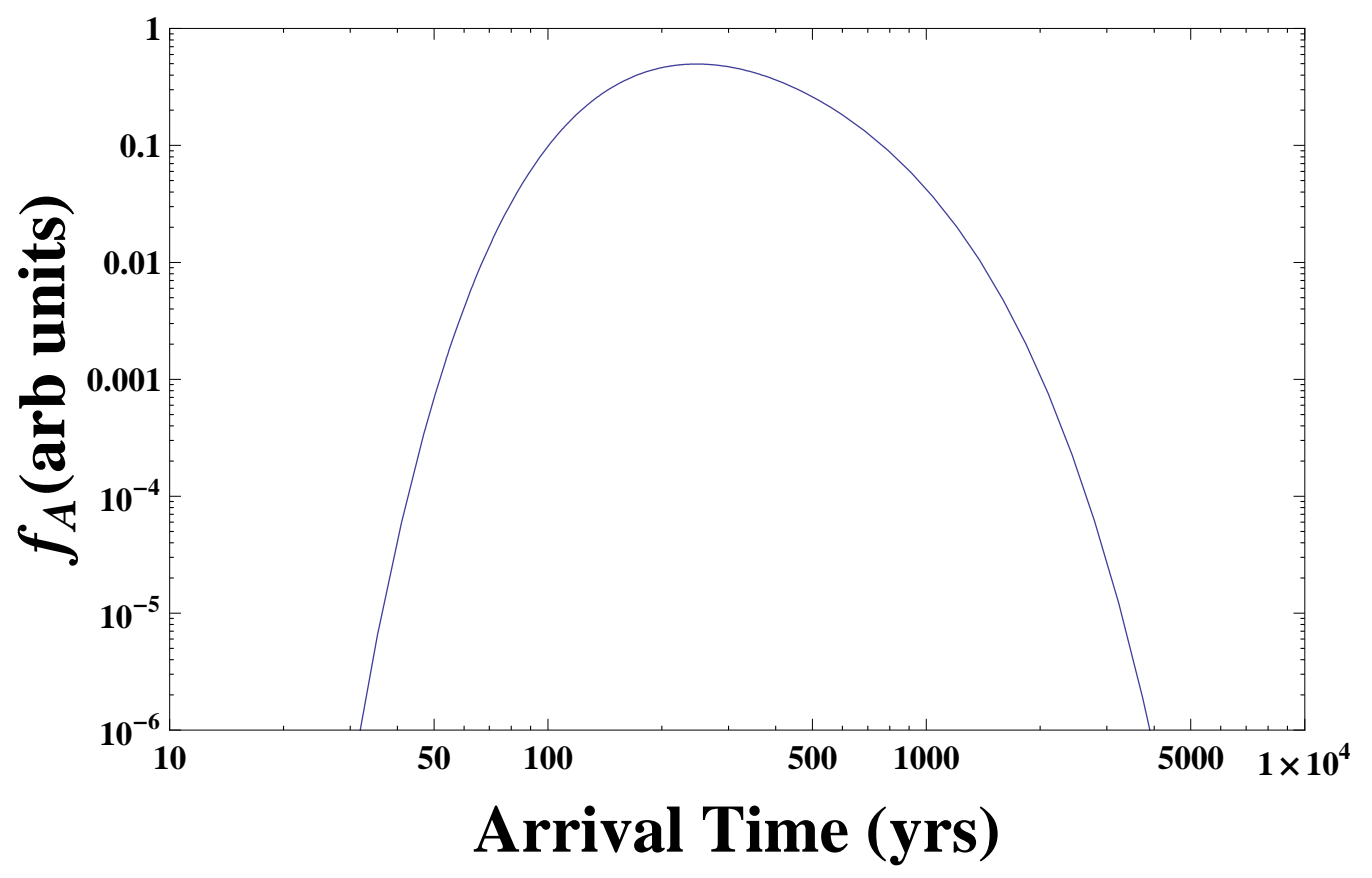

Figure 3: Arrival time probability distribution in arbitrary units for primary cosmic rays including only delays at the source. A cosmic ray source of $10^{10} \mathrm{GeV}$ protons was used in a source $100 \mathrm{kpc}$ wide with $B=10^{-6} \mathrm{G}$ and $l_{c}=0.1 \mathrm{kpc}$.

of the source by a factor of

$$
f_{\text {damp }} \sim N_{\text {active }} \frac{t_{\text {active }}}{t_{\text {delay }}},
$$

where $t_{\text {delay }}$ is the typical proton delay at the source, $t_{\text {active }}$ is the typical time the source is active or flaring and $N_{\text {active }}$ is the number of times the source is active in the time period $t_{\text {delay }}$. This damping will not be a significant effect, especially since the typical deflections at the source will not be enough to affect the beaming factors.

Using Eq. (3) and these typical time delays we see that the model independent lower limit of $10^{-17} \mathrm{G}$ would remain unchanged and the upper limit of $3 \times 10^{-14} \mathrm{G}$ would be lowered by roughly one order of magnitude. Thus the limits we provided for cosmic rays are still the most conservative limits 
even including all timing uncertainties. Thus, for the case of cosmic rays dominating the signal, we can report a model-independent 95\% CL interval for the IGMF of

$$
1 \times 10^{-17} \mathrm{G}<\mathrm{B}<3 \times 10^{-14} \mathrm{G} .
$$

Our results represent the first 95\% confidence interval measurements of the IGMF based on the spectral data alone. The method used here to probe the IGMF will become more powerful in the future. Future observations of distant blazars will not only improve the limits on the IGMF, but also constrain AGN and EBL models. The measured values of IGMFs can be used to distinguish between the cosmological and astrophysical scenarios for the origin of all astrophysical magnetic fields. In addition, this information will lead to a better understanding of gamma rays and cosmic rays, as well as the properties of AGN and of universal photon backgrounds.

The authors thank F. Aharonian, J. Beacom, P. Blasi, C. Dermer, S. Razzaque, and E. Waxman for helpful comments. The work of W.E. and A.K. was supported by DOE grant DE-FG03-91ER40662 and NASA ATP grant NNX08AL48G. The work of S.A. was supported by Japan Society for Promotion of Science.

\section{References}

[1] P. P. Kronberg, Rept. Prog. Phys. 57 (1994) 325; A. Kandus, K. E. Kunze and C. G. Tsagas, arXiv:1007.3891.

[2] J. D. Barrow, P. G. Ferreira and J. Silk, Phys. Rev. Lett. 78 (1997) 3610; K. Subramanian and J. D. Barrow, Phys. Rev. Lett. 81 (1998) 3575; P. Blasi, S. Burles and A. V. Olinto, Astrophys. J. 514 (1999) L79; K. Jedamzik, V. Katalinic and A. V. Olinto, Phys. Rev. Lett. 85 (2000) 700 .

[3] R. Plaga, Nature, 374 (1995) 430.

[4] F. A. Aharonian, P. S. Coppi, H. J. Volk, Astrophys. J. 423, L5-L8 (1994).

[5] S. Ando and A. Kusenko, Astrophys. J. 722 (2010) L39; A. Neronov et al., Astron. and Astrophys., 526 (2010) 90.

[6] A. Neronov, I. Vovk, Science 328 (2010) 73. 
[7] F. Tavecchio et al., Mon. Not. Roy. Astron. Soc. 406 (2010) L70.

[8] K. Dolag et al., Astrophys. J. 727 (2011) L4.

[9] C. D. Dermer et al., arXiv:1011.6660].

[10] W. Essey and A. Kusenko, Astropart. Phys. 33 (2010) 81.

[11] W. Essey, O. E. Kalashev, A. Kusenko and J. F. Beacom, Phys. Rev. Lett. 104 (2010) 141102.

[12] W. Essey, O. Kalashev, A. Kusenko and J. F. Beacom, Astrophys. J., 731 (2011) 51.

[13] P. L. Biermann et al., Int. J. Mod. Phys. D 18 (2009) 1577; arXiv:1012.0204 [astro-ph.GA].

[14] For recent reviews, see, e.g., T. Stanev, Mod. Phys. Lett. A, 25, (2010) 1467; T. K. Gaisser, arXiv:1010.5996 [astro-ph.HE].

[15] K. Dolag et al., JCAP 0501 (2005) 009; D. Ryu, H. Kang, J. Cho, S. Das, Science, 320 (2008) 909.

[16] F. Aharonian et al., Astron. Astrophys. 475 (2007) L9.

[17] F. Aharonian et al., Astron. Astrophys. 470 (2007) 475.

[18] F. Aharonian et al., Astron. Astrophys. 473 (2007) L25.

[19] A. Zech et al., in Proceedings of 31st ICRC (Lodz), 2009.

[20] J. S. Perkins and VERITAS Collaboration, Bulletin of the American Astronomical Society,42 (2010) 708.

[21] C. Xu et al., Astrophys. J., 562 (2001) 179.

[22] F. W. Stecker, M. A. Malkan and S. T. Scully, Astrophys. J. 648 (2006) 774 .

[23] M. H. Salamon and F. W. Stecker, Astrophys. J. 493 (1998) 547; T. M. Kneiske et al., Astron. Astrophys. 386(2002) 1; ibid., 413 (2004) 807; A. Franceschini, G. Rodighiero and M. Vaccari, Astron. Astrophys. 487 (2008) 837; S. Horiuchi, J. F. Beacom and E. Dwek, Phys. Rev. D 
79 (2009) 083013; J. R. Primack, R. C. Gilmore and R. S. Somerville, AIP Conf. Proc. 1085 (2009) 71; R. C. Gilmore et al., MNRAS, 399 (2009) 1694; S. Razzaque, C. D. Dermer and J. D. Finke, Astrophys. J. 697 (2009) 483; J. D. Finke, S. Razzaque and C. D. Dermer, Astrophys. J. 712 (2010) 238.

[24] A. A. Abdo et al., Astrophys. J. 723 (2010) 1082.

[25] J. A. Peacock, MNRAS 196 (1981) 135; J. G. Kirk and P. Schneider, Astrophys. J., 315 (1987) 425; A. F. Heavens and L. O. Drury, MNRAS 235 (1988) 997; J. Bednarz and M. Ostrowski, Phys. Rev. Lett. 80 (1998) 3911; M. A. Malkov and L. O'C Drury, Reports Prog. Phys., 64 (2001) 429.

[26] F. W. Stecker, M. G. Baring and E. J. Summerlin, Astrophys. J. 667 (2007) L29; F. Aharonian, D. Khangulyan and L. Costamante, MNRAS, 387, (2008) 1206.

[27] A. Neronov and D. V. Semikoz, Phys. Rev. D 80 (2009) 123012. 